INPLASY

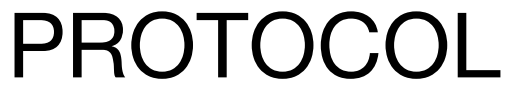

To cite: Wang et al. Long-term outcomes of single stenting compared with double stenting strategy for unprotected left main coronary artery disease: a protocol for systematic review and meta-analysis. Inplasy protocol 2020110030. doi:

10.37766/inplasy2020.11.0030

Received: 08 November 2020

Published: 08 November 2020

Corresponding author:

Zheng Zhang

zhangzhengldyy@126.com

Author Affiliation:

Department of Cardiology, the first hospital of Lanzhou University, Lanzhou, China.

Support: None.

Review Stage at time of this submission: Preliminary searches.

Conflicts of interest: None.

\section{Long-term outcomes of single stenting compared with double stenting strategy for unprotected left main coronary artery disease: a protocol for systematic review and meta-analysis}

Wang, JJ1; Li, X²; Yan, DD³ Zhang, Z4.

Review question / Objective: Population: unprotected left main coronary artery disease; Intervention: single stenting strategy; Comparator: double stenting strategy; Outcome: major adverse cardiovascular events (MACE), all-cause mortality, cardiac mortality, myocardial infarction, target lesion revascularization (TLR); Study: RCT, cohort study. Information sources: A systematic search of online databases including PubMed, EMBASE, Web of science and Cochrane Library will be performed until the end of September 2020 using the keywords "unprotected left main coronary artery disease"," double stenting strategy" and "drug-eluting stent". In addition, congress and conference proceedings will be manually retrieved. Related articles and references of included research will also be tracked to find potential studies. If significant data was incomplete in included study, we will contact the authors to get unpublished data.

INPLASY registration number: This protocol was registered with the International Platform of Registered Systematic Review and Meta-Analysis Protocols (INPLASY) on 08 November 2020 and was last updated on 08 November 2020 (registration number INPLASY2020110030).

\section{INTRODUCTION}

Review question / Objective: Population: unprotected left main coronary artery disease; Intervention: single stenting strategy; Comparator: double stenting strategy; Outcome: major adverse cardiovascular events (MACE), all-cause mortality, cardiac mortality, myocardial infarction, target lesion revascularization (TLR); Study: RCT, cohort study.

Condition being studied: Unprotected left main coronary artery (ULMCA) disease 
defined as left main coronary artery disease without right to left collateral circulation or bridging blood supply is more dangerous and the most difficult problem for clinical interventionists, so the choice of treatment methods and strategies is very important. Coronary artery bypass grafting (CABG) has been recommended as the gold standard for ULMCA disease. However, with the development of percutaneous coronary intervention (PCI) techniques and the emergence of drugeluting stents (DES), $\mathrm{PCl}$ has become a minimally invasive and therapeutic approach comparable to CABG intervention. Although the single stent (SS) approach of implanting one stent in the main branch only is a default intervention strategy for ULMCA lesions, the double stent (DS) approach of implanting stents in both the main branch and side branch is still used in patients with severely diseased side branches. Only one studies are available comparing SS versus DS strategy on the short-term clinical outcomes of ULMCA disease.Given the scarcity of longterm data with either strategy, we use hazard ratios (HRs) to perform the first meta-analysis to assess the long-term effect of SS versus DS for intervention of ULMCA disease in the DES era.

\section{METHODS}

Participant or population: ULMCA disease was diagnosed by coronary angiography, requiring percutaneous coronary interventions. Patient $\geq 18$ years old. No restrictions on gender or age, but Pregnant women and Cardiogenic shock excluded.

Intervention: Single stenting strategy: implanting one stent in the main branch only.

Comparator: Double stenting strategy: implanting stents in both the main branch and side branch and side branch.

Study designs to be included: RCT or cohort study.

Eligibility criteria: Randomised controlled trial (RCT) and cohort study with long-term follow up defined as longer than $\mathbf{1 2}$ months after the completion of the intervention.

Information sources: A systematic search of online databases including PubMed, EMBASE, Web of science and Cochrane Library will be performed until the end of September 2020 using the keywords "unprotected left main coronary artery disease"," double stenting strategy" and "drug-eluting stent". In addition, congress and conference proceedings will be manually retrieved. Related articles and references of included research will also be tracked to find potential studies. If significant data was incomplete in included study, we will contact the authors to get unpublished data.

Main outcome(s): Major adverse cardiovascular events (MACE).

Additional outcome(s): All-cause mortality, cardiac mortality, myocardial infarction, target lesion revascularization.

Data management: Two review authors will screen the titles and abstracts of studies identified by the search. The two authors will conduct full text screening on all studies identified by either author as possibly suitable for inclusion. and determine eligibility for inclusion. Disagreements will be referred to a third author. A data extraction form will be developed and data extraction will be completed by two authors. Data extracted will include author, year, study type, number of participants, intervention, control, demographics, procedure data, stent type, classification of double stent techniques, the incidence of major adverse cardiovascular events, all-cause mortality, cardiac mortality, myocardial infarction, target lesion revascularization.

Quality assessment / Risk of bias analysis: Included study bias will be independently assessed by two reviewers and any disagreement will be solved by a third reviewer. For randomized controlled trials, we will use the Cochrane risk of bias tools to evaluate potential bias in seven specific domains: (1) sequence generation, (2) 
allocation concealment, (3) blinding of participants and personnel, (4) blinding of outcome assessment, (5) incomplete outcome data, (6)selective outcome reporting, (7) other bias. For propensitymatch cohort studies, 9-star NewcastleOttawa Scale will be applied, which rates studies based on eight criteria in three sources of bias.

Strategy of data synthesis: Participants will be divided into two groups: single stent strategy and double stent strategy. Data synthesis and analyses were performed using Stata. Studies use hazard ratios of Cox proportional hazard models. We will use hazard ratios to approximate the relative risk. Furthermore, potential publication bias will be assessed using funnel plots. Heterogeneity will be assessed among included studies both qualitatively (by comparing the characteristics of included studies) and quantitatively (using the $X^{2}$ test of heterogeneity and $I^{2}$ statistic). We performed meta-analyses by using random effects models. When an $I^{2}$ score of $>50 \%$ was obtained, heterogeneity was considered to be substantial and subgroup analysis was performed.

Subgroup analysis: We will also conduct subgroup analysis to find more potential information based on pre-set criteria in different follow-up time.

Sensibility analysis: If the heterogeneity is high, we will conduct sensitivity analyses based on the follow-up time.

\section{Country(ies) involved: China.}

Keywords: Unprotected left main coronary artery disease; single and double stenting strategy; drug-eluting stent; long-term clinical outcomes.

Contributions of each author:

Author 1 - Jia-jie Wang - The author conceived the idea for this study, designed the meta-analysis, provided statistical advice and input, drafted the protocol and reviewed the protocol and provided critical feedback.

Author 2 - Xin Li - The author designed the meta-analysis, drafted the protocol.

Author 3 - Dong-dong Yan - The author provided statistical advice and input, reviewed the protocol and provided critical feedback.

Author 4 - Zheng Zhang - The author conceived the idea for this study. 